\title{
Intestinal obstruction following TAPP a rare complication
}

\begin{abstract}
The development of newer techniques in the field of surgery is associated with its own set of possible complications. Laparoscopic mesh repair is now widely accepted as the standard of care for inguinal hernias. Both transabdominal preperitoneal approach and totally extra peritoneal approach are almost equally popular worldwide. Laparoscopic hernia repair is a frequently performed operation in our hospital. Although it has many advantages over open inguinal hernia repair, laparoscopic surgery is not without complications. Small bowel obstruction is a complication unique to laparoscopic repair of inguinal hernias. It is reported following transabdominal preperitoneal repairs. We present a case of small bowel incarceration through a peritoneal defect after a totally extraperitoneal inguinal hernia repair.
\end{abstract}

Keywords: transabdominal preperitoneal, blood count, random blood sugar, serum creatinine, prothrombin time, chest X-ray, electrocardiogram
Volume II Issue I - 2020

\author{
Faris Dawood Al-Aswad ${ }^{1,2}$ \\ 'Department of General Surgeon, University of Baghdad, Iraq \\ ${ }^{2} \mathrm{NMC}$ Specialty Hospital, UAE
}

Correspondence: Faris Dawood Al-Aswad, Department of
General Surgeon, University of Baghdad, Iraq, NMC Speciality
Hospital, P.O.Box 7832, Dubai, UAE, Tel 0097I-509523829, Fax 0097I-4 2678889 Email faris_alaswad@yahoo.com

Received: December 12, 2019 | Published: January 20, 2020

\section{Case report}

A 40-year-old male patient reported to our outpatient department with complaints of left inguinal swelling noted since 12 weeks. He had associated complaints of occasional dragging pain on the left side. There were no associated co morbidities; he was operated before more than 2years as case of open left varicocele. The patient had a body mass index of 24.45 (height $168 \mathrm{~cm}$ and weight $69 \mathrm{~kg}$ ). His vitals were stable and systemic examination did not reveal any abnormality. On clinical examination, he had a left inguinal hernia. The routine pre-operative investigations including complete blood count, random blood sugar, serum creatinine, prothrombin time, chest X-ray, and electrocardiogram were within normal limits.

Accordingly, the patient was admitted to our hospital on May 2019. Under general anesthesia, pneumoperitoneum was created through umbilicus using Hassons method. A $10 \mathrm{~mm}$ port was placed in the infraumbilical region and 2 ports of $5 \mathrm{~mm}$ each on either side at the level of the umbilicus. The preperitoneal space was created with monopolar cautery, and blunt dissection on the left side hernial sac was reduced. A $10 \mathrm{~cm} \times 15 \mathrm{~cm} 3$ D BARD mesh was introduced and properly unrolled. It was then anchored using $5 \mathrm{~mm}$ tackers (ProtackTM AutoSutureTM Fixation device) to Cooper's ligament, at the upper medical corner of the Hasselbach's triangle, and also at the upper lateral aspect above the level of the anterior superior iliac spine. The peritoneal flap was closed with running 2.0 Vicryl suture. The ports were closed with subcuticular sutures. At the time of creating the peritoneal flap a hole was created in it and it was closed with bi polar cautery at the end of surgery after mesh replacement.

The patient had an uneventful post-operative period and was discharged the next day on routine oral analgesics (tablet diclofenac $50 \mathrm{mg}$ with tablet paracetamol $50 \mathrm{mg}$ ) twice daily for days and as required. Routine follow-up after 4days was normal.

The patient presented again in the emergency after 7days from discharging from hospital, with complaints of abdominal distension and the inability to pass flatus and motion for 2days. This was associated with multiple episodes of vomiting. Clinical examination showed a distended abdomen with generalized mild tenderness and increased bowel sounds. X-ray showed multiple air-fluid levels. Routine laboratory investigations were within normal limits. The patient was initially managed conservatively with nil per oral, nasogastric tube suction, intravenous fluids intravenous antibiotics and other supportive management for $48 \mathrm{~h}$. However, in view of the persistence of intestinal obstruction, a decision to interfere surgically was made.

Contrast-enhanced $\mathrm{CT}$ was not done as the condition was very obvious clinically. He was taken up for diagnostic laparoscopy under general anesthesia which showed herniation of distal small bowel loop through a peritoneal defect of the previous hernioplasty site with dense adhesions of the bowel to the mesh. The herniated bowel was reduced, the hernia defect was dealt with closure by direct stitching and glue again of the mesh to the abdominal wall after aspiration of the reactionary fluid, stitching itself was not an easy task because of the friability and thinness of the peritoneal flap and this is why a glue is used. The patient had an uneventful recovery and was discharged on the fourth post-operative day. The patient underwent follow-up examination 21 days after discharge and did not have any significant abnormality. ${ }^{1-3}$

\section{Discussion}

Laparoscopic repair has gained universal acceptance and is considered the standard of care for bilateral and recurrent inguinal hernias. TAPP mesh hernioplasty is more widely performed than totally extraperitoneal (TEP) mesh hernioplasty. ${ }^{4}$ The significant drawback of TEP approach is the lack of reproducibility by different surgeons with comparable results. However, it is gaining popularity recently. The frequent complication encountered after surgery include hematoma, seroma neuralgia, chronic pain, and recurrences. Rare complications include mesh erosions into bladder and mesh infections. 
Small bowel obstructions have been reported after laparoscopic repair due to herniation of bowel loop through the port site or through a defect in peritoneal closure A literature review showed about 16 cases of intestinal obstruction due to herniation through the peritoneal defect similar to our case requiring operative intervention. ${ }^{4}$

Kapiris et al. ${ }^{5}$ in their 7 -year study of 3017 patients undergoing TAPP hernia repair had $7(0.23 \%)$ cases of small bowel obstruction due to herniation through the peritoneal defect. In the initial part of the study, they performed a stapled peritoneal closure, but later sutured closure was performed. They observed the decreased incidence of this complication following sutured peritoneal closure. Phillips et al. ${ }^{6}$ assessed complications following 3229 laparoscopic hernia repairs of which $1944(60 \%)$ were TAPP mesh hernioplasty for inguinal hernias. There were $4(0.20 \%)$ cases of small bowel obstruction due to herniation through peritoneal defect or port site.

Ohta et al., ${ }^{7}$ Tsang et al. ${ }^{8}$ and Cueto et al. ${ }^{9}$ each reported one case of intestinal herniation through a peritoneal defect in the early postoperative period, all of which was managed laparoscopically. Another interesting case report by Köhleret al. ${ }^{10}$ of intestinal obstruction following self-anchoring barbed suture device peritoneal closure deserves to be noted.

In our case, the patient had a sutured closure of the peritoneal flap after a laparoscopic TAPP mesh hernioplasty. He presented 7days after the surgery with features of intestinal obstruction. The herniated loop of bowel through the peritoneal defect was densely adherent to the mesh and it was managed again laprascopically. The Guidelines of International Endo Hernia society ${ }^{8}$ recommends thorough closure of the peritoneal defect in TAPP to decrease the risk of bowel obstruction (Level 3 Grade C) and running absorbable suture as an appropriate method to close the defect (Level 5 Grade D) (Figures 1-5). ${ }^{9}$

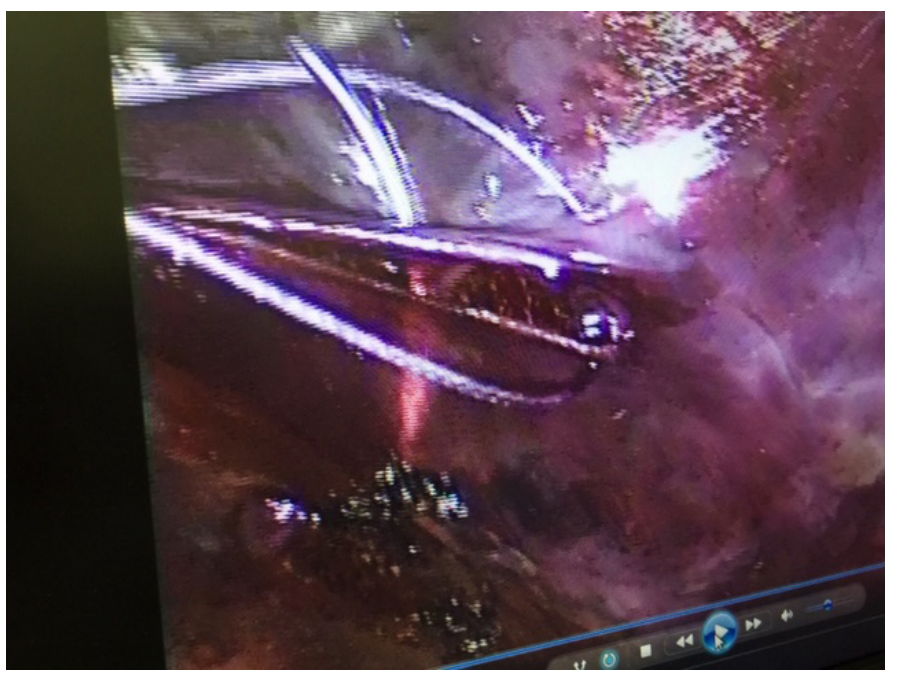

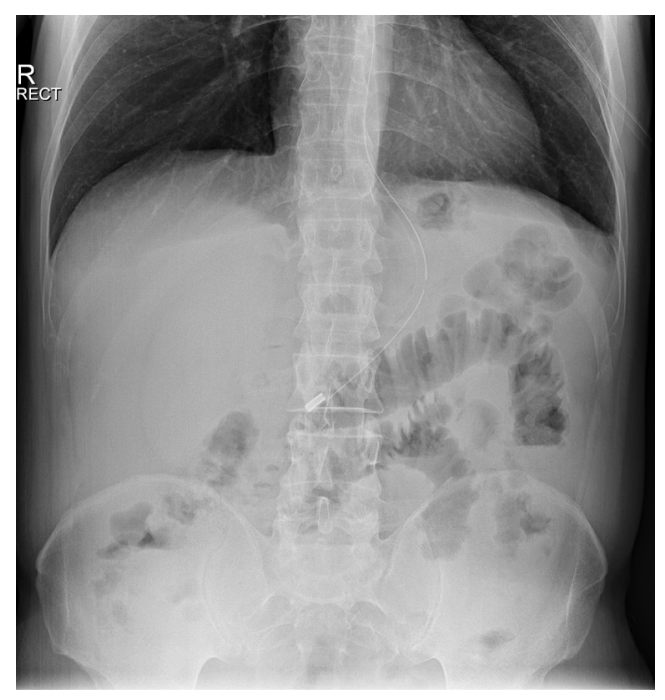

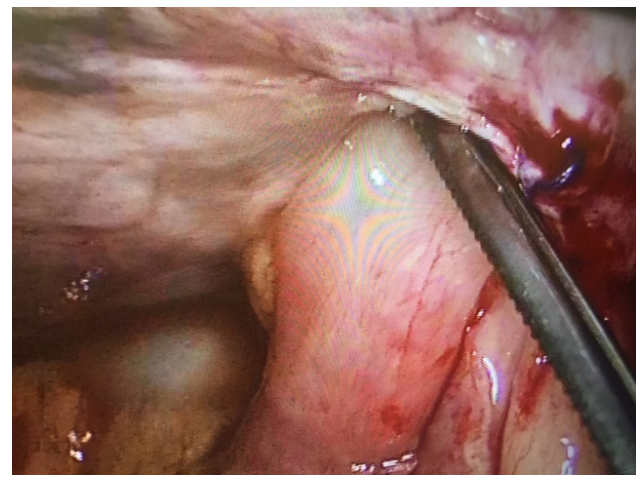

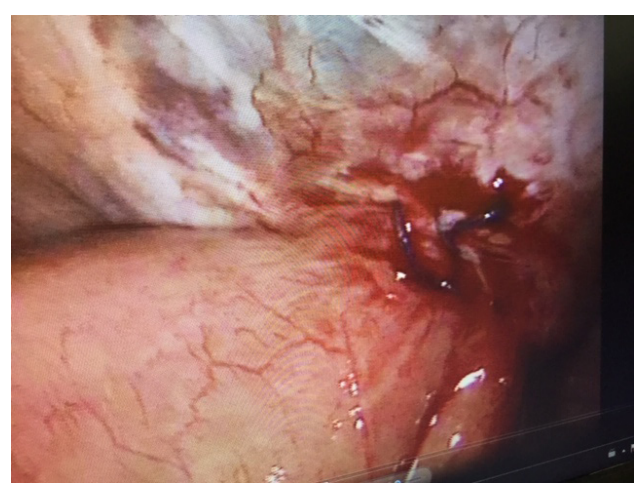

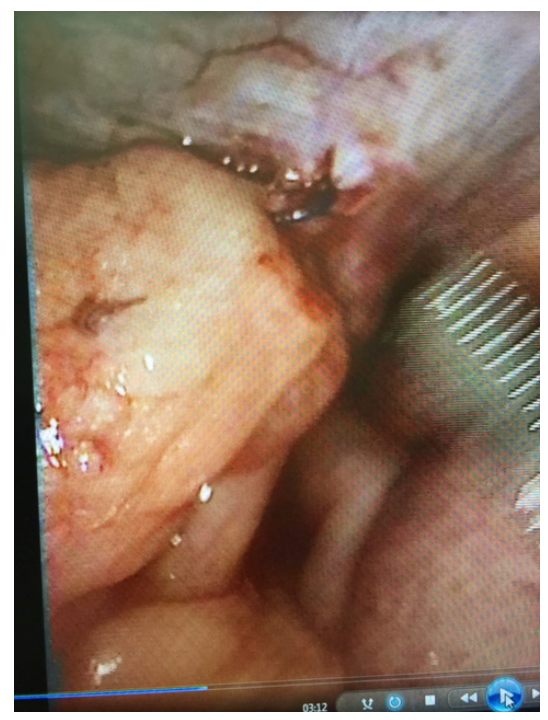




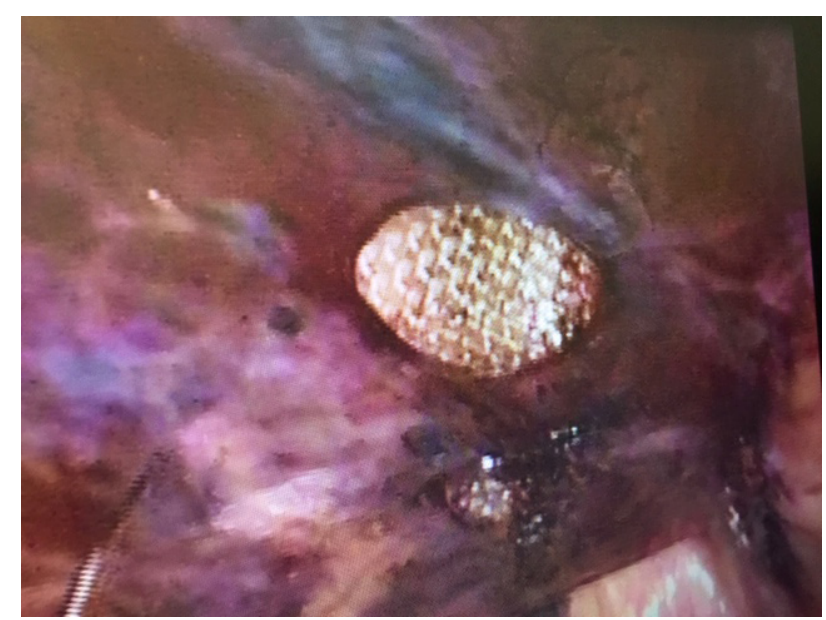

\section{Conclusion}

Although laparoscopic TAPP mesh hernioplasty has gained widespread popularity and acceptance for management of inguinal hernias, it must be remembered that complications may occur, some of them though rare, can cause significant morbidity. An adequate and proper peritoneal closure is recommended to reduce the risk of bowel herniation and intestinal obstruction. We prefer to perform a diagnostic laparoscopy in case of the complication, but conversion to laparotomy may be required.

\section{Acknowledgments}

None.

\section{Conflicts of interest}

Author declares that there are no conflicts of interest towards publication of this article.

\section{Funding}

None.

\section{References}

1. Tsang S, Normand R, Karlin R. Small bowel obstruction: A morbid complication after laparoscopic herniorrhaphy. Am Surg. 1994;60(5):332-334.

2. Cueto J, Vázquez JA, Solís MA, et al. Bowel obstruction in the postoperative period of laparoscopic inguinal hernia repair (TAPP): Review of the literature. JSLS. 1998;2(3):277-280.

3. Köhler G, Mayer F, Lechner M. Small bowel obstruction after TAPP repair caused by a self-anchoring barbed suture device for peritoneal closure: Case report and review of the literature. Hernia. 2015;19(3):389-394.

4. Bittner R, Arregui ME, Bisgaard T, et al. Guidelines for laparoscopic (TAPP) and endoscopic (TEP) treatment Kingsnorth A, LeBlanc K. Hernias: Inguinal and incisional. Lancet. 2003;362:1561-171.

5. Payne JH Jr, Grininger LM, Izawa MT, et al. Laparoscopic or open inguinal herniorrhaphy? A randomized prospective trial. Arch Surg. 1994;129(9):973-979.

6. EU Hernia Trialists Collaboration. Laparoscopic compared with open methods of groin hernia repair: Systematic review of randomized controlled trials. Br J Surg. 2000;87(7):860-867.

7. Sayad R, Hallak A, Ferzli G. Laparoscopic herniorrhaphy: Review of complications and recurrence. J Laparoendosc Adv Surg Tech A. 1998;8(1):3-10.

8. Kapiris SA, Brough WA, Royston CM, et al. Laparoscopic transabdominal preperitoneal (TAPP) hernia repair: A 7-year twocenter experience in 3017 patients. Surg Endosc. 2001;15(9):972-925.

9. Phillips EH, Arregui M, Carroll BJ, et al. Incidence of complications following laparoscopic hernioplasty. Surg Endosc. 1995;9(1):16-21. 